\title{
(2+1)-Dimensional Davey-Stewartson II Equation for a Two-Dimensional Nonlinear Monatomic Lattice
}

\author{
Zhi-Fang Li ${ }^{\mathrm{a}}$ and Hang-Yu Ruan ${ }^{\mathrm{a}, \mathrm{b}, \mathrm{c}}$ \\ a Department of Physics, Ningbo University, Ningbo 315211, China \\ b Nonlinear Science Center, Ningbo University, Ningbo 315211, China \\ c State Key Laboratory of Scientific and Engineering Computing, Institute of Computational \\ Mathematics and Scientific Engineering Computing, Academy of Mathematics and System \\ Sciences, the Chinese Academy of Sciences, P. O. Box 2719, Beijing 100080, China
}

Reprint requests to H.-Y.R.; E-mail: hyruan@mail.nbip.net

Z. Naturforsch. 61a, 45 - 52 (2006); received October 12, 2005

A two-dimensional monatomic lattice with nearest-neighbor interaction is investigated by the method of multiple scales combined with a quasidiscreteness approximation. The Davey-Stewartson II equation (DS-II) is obtained from the original two-dimensional (2D) differential-difference system. By solving the DS-II, explicit periodic solutions, soliton solutions and rational function solutions are obtained, and the leading order approximated solutions of the 2D monatomic lattice are constructed by explicit solutions of the DS-II.

Key words: Two-Dimensional Monatomic Lattice; Method of Multiple Scales; Quasi-Discreteness Approximation; Davey-Stewartson II Equation.

\section{Introduction}

In recent decades there has been a great development in soliton formation and its application in onedimensional (1D) systems. The observation of soliton modes in energy propagating along a biological molecule chain has attracted more and more interest. New achievements in studying topological solitons in polyacetylene chains and the phenomena of transformation with the action of an electric field have been obtained [1]. The electric field soliton [2], optical soliton [3], breather [4], and so on are interesting problems. Since the pioneering work of Fermi, Pasta, and Ulam [5] and of Zabusky and Kruskal [6] on nonlinear dynamics in a lattice, a great variety of researches on the dynamical localization in discrete systems has been stimulated, especially on one-dimensional lattice solitons, which are localized nonlinear excitations due to the balance between nonlinearity and dispersion [7]. The method of multiple scales is an effective method, and by use of it one can reduce the lattice system, which is a set of differential-difference equations and can hardly be solved exactly, to a set of partial differential equations for a slowly changing envelope from which one can get explicit solutions, such as a nonlinear Schrödinger (NLS) equation [8] and a Korteweg-de Vries (KdV) equation [9]. Basing on 1D

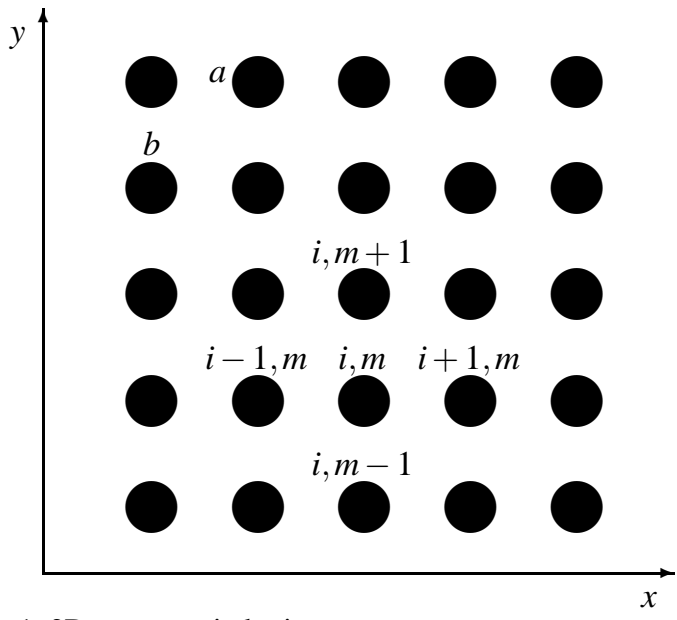

Fig. 1. 2D monatomic lattice.

nonlinear effects, people have also made many investigations on soliton equations of two-dimensional (2D) systems $[10-15]$.

The present paper is devoted to investigate the equations of nonlinear vibration in a two-dimensional discrete monatomic lattice with nearest-neighbor interaction, which is shown in Fig. 1, where $a$ and $b$ are lattice constants. When higher power terms with weak nonlinear interaction are neglected, the Hamiltonian of the system is 


$$
\begin{aligned}
H= & \sum_{l, m}\left[\frac{p_{l, m}^{2}}{2 M}+\frac{1}{2} K_{2 x}\left(u_{l+1, m}-u_{l, m}\right)^{2}+\frac{1}{3} K_{3 x}\left(u_{l+1, m}-u_{n, m}\right)^{3}+\frac{1}{4} K_{4 x}\left(u_{l+1, m}-u_{l, m}\right)^{4}\right] \\
& +\sum_{l, m}\left[\frac{1}{2} K_{2 y}\left(u_{l, m+1}-u_{l, m}\right)^{2}+\frac{1}{3} K_{3 y}\left(u_{l, m+1}-u_{l, m}\right)^{3}+\frac{1}{4} K_{4 y}\left(u_{l, m+1}-u_{l, m}\right)^{4}\right],
\end{aligned}
$$

where $u_{l, m}(t)$ is the displacement of an atom with mass $M$ from its equilibrium position, and $K_{2 x}, K_{3 x}, K_{4 x}$ and $K_{2 y}, K_{3 y}, K_{4 y}$ are harmonic, cubic, and biquadratic force constants in the $x$ - and $y$-direction, respectively. By using the method of multiple scales $[8,16-$ 19] combined with a quasidiscreteness approximation [20], the Davey-Stewartson II equation (DS-II) is obtained from the original 2D lattice. The explicit solutions of the DS-II are given, and then the leading order approximated solutions are constructed by the explicit solutions of DS-II.

The organization of the paper is as follows. In Section 2, the $(2+1)$-dimensional DS-II is derived from the original 2D monatomic lattice. Section 3 is devoted to solve the DS-II and obtain the explicit solutions which are used to construct the leading order approximated solutions of the original 2D lattice. The last section contains a short conclusion.

\section{Asymptotic Expansion and the (2+1)-Dimen- sional Davey-Stewartson II Equation}

According to (1), the nonlinear vibration equation for $u_{l, m}$ has the form

$$
\begin{aligned}
u_{l, m}= & J_{2 x}\left(u_{l+1, m}-2 u_{l, m}+u_{l-1, m}\right) \\
& +J_{3 x}\left[\left(u_{l+1, m}-u_{l, m}\right)^{2}-\left(u_{l-1, m}-u_{l, m}\right)^{2}\right] \\
& +J_{4 x}\left[\left(u_{l+1, m}-u_{l, m}\right)^{3}+\left(u_{l-1, m}-u_{l, m}\right)^{3}\right] \\
& +J_{2 y}\left(u_{l, m+1}-2 u_{l, m}+u_{l, m-1}\right) \\
& +J_{3 y}\left[\left(u_{l, m+1}-u_{l, m}\right)^{2}-\left(u_{l, m-1}-u_{l, m}\right)^{2}\right] \\
& +J_{4 y}\left[\left(u_{l, m+1}-u_{l, m}\right)^{3}+\left(u_{l, m-1}-u_{l, m}\right)^{3}\right],
\end{aligned}
$$

where $J_{2 x}=\frac{K_{2 x}}{M}, J_{3 x}=\frac{K_{3 x}}{M}, J_{4 x}=\frac{K_{4 x}}{M}$ and $J_{2 y}=\frac{K_{2 y}}{M}$,

$J_{3 y}=\frac{K_{3 y}}{M}, J_{4 y}=\frac{K_{4 y}}{M}$. In order to include the effects of the anharmonicity and discreteness of the lattice, we use the method of multiple scales [8,16-19] and a quasidiscreteness approximation [20] to obtain the equations which describe the development of the modulation of the amplitude in the lowest order of an asymptotic expansion. In this treatment one sets

$$
\begin{aligned}
u_{l, m}(t)= & \varepsilon u^{(1)}\left(\xi_{l}, \eta_{m}, \theta_{l}, \tau\right)+\varepsilon^{2} u^{(2)}\left(\xi_{l}, \eta_{m}, \theta_{l}, \tau\right) \\
& +\varepsilon^{3} u^{(3)}\left(\xi_{l}, \eta_{m}, \theta_{l}, \tau\right)+\ldots \\
= & \sum_{j=1}^{\infty} \varepsilon^{j} u_{l, m, l}^{(j)}
\end{aligned}
$$

where $\varepsilon$ is a small but finite parameter denoting the relative amplitude of the excitations and $u_{l, m, l}^{(j)}=$ $u^{(j)}\left(\xi_{l}, \eta_{m}, \theta_{l}, \tau\right), \xi_{l}, \eta_{m}$ and $\tau$ are "slow" variables, respectively, defined by $\xi_{l}=\varepsilon(l a-\lambda t), \eta_{m}=\varepsilon m b$ and $\tau=\varepsilon^{2} t$. They are called multiple scales variables. The parameter $\lambda$ is to be determined later. The "fast" variable, $\theta_{l}=k l a-\omega t$, represents the phase of the carrier wave. Here $k$ and $\omega$ are the wave number and frequency of the carrier wave, respectively. In term of these notations, by substituting (3) into (2) and comparing the powers of $\varepsilon$, one obtains the following equations:

$$
\begin{gathered}
\frac{\partial^{2}}{\partial t^{2}} u_{l, m, l}^{(j)}-J_{2 x}\left(u_{l, m, l+1}^{(j)}-2 u_{l, m, l}^{(j)}+u_{l, m, l-1}^{(j)}\right) \\
=f_{l, m, l}^{(j)}, \quad j=1,2,3, \ldots
\end{gathered}
$$

with

$$
f_{l, m, l}^{(1)}=0,
$$

$$
\begin{aligned}
f_{l, m, l}^{(2)}= & 2 \lambda \frac{\partial^{2}}{\partial t \partial \xi_{l}} u_{l, m, l}^{(1)}+J_{2 x} a \frac{\partial}{\partial \xi_{l}}\left(u_{l, m, l+1}^{(1)}-u_{l, m, l-1}^{(1)}\right)+J_{3 x}\left[\left(u_{l, m, l+1}^{(1)}-u_{l, m, l}^{(1)}\right)^{2}-\left(u_{l, m, l-1}^{(1)}-u_{l, m, l}^{(1)}\right)^{2}\right],(6) \\
f_{l, m, l}^{(3)}= & 2 \lambda \frac{\partial^{2}}{\partial t \partial \xi_{l}} u_{l, m, l}^{(2)}+J_{2 x} a \frac{\partial}{\partial \xi_{l}}\left(u_{l, m, l+1}^{(2)}-u_{l, m, l-1}^{(2)}\right)+2 a J_{3 x} \frac{\partial u_{l, m, l+1}^{(1)}}{\partial \xi_{l}}\left(u_{l, m, l+1}^{(1)}-u_{l, m, l}^{(1)}\right) \\
& +2 a J_{3 x} \frac{\partial}{\partial \xi_{l}} u_{l, m, l-1}^{(1)}\left(u_{l, m, l-1}^{(1)}-u_{l, m, l}^{(1)}\right)-2 \frac{\partial^{2}}{\partial t \partial \tau} u_{l, m, l}^{(1)}-\lambda^{2} \frac{\partial^{2}}{\partial \xi_{l}^{2}} u_{l, m, l}^{(1)}+\frac{1}{2} J_{2 x} a^{2} \frac{\partial^{2}}{\partial \xi_{l}^{2}}\left(u_{l, m, l+1}^{(1)}+u_{l, m, l-1}^{(1)}\right)
\end{aligned}
$$




$$
\begin{aligned}
&+J_{2 y} a^{2} \frac{\partial^{2}}{\partial \eta_{m}^{2}} u_{l, m, l}^{(1)}+2 J_{3 x}[ u_{l, m, l-1}^{(2)}\left(u_{l, m, l}^{(1)}-u_{l, m, l-1}^{(1)}\right)+u_{l, m, l}^{(2)}\left(u_{l, m, l-1}^{(1)}-u_{l, m, l+1}^{(1)}\right) \\
&+\left.u_{l, m, l+1}^{(2)}\left(u_{l, m, l-1}^{(1)}-u_{l, m, l+1}^{(1)}\right)\right] \\
&+J_{4 x}\left(u_{l, m, l+1}^{(1)}-2 u_{l, m, l}^{(1)}+u_{l, m, l-1}^{(1)}\right)\left[u_{l, m, l+1}^{(1)}\left(u_{l, m, l+1}^{(1)}-u_{l, m, l}^{(1)}\right)+u_{l, m, l}^{(1)}\left(u_{l, m, l}^{(1)}-u_{l, m, l-1}^{(1)}\right)\right. \\
&\left.+u_{l, m, l-1}^{(1)}\left(u_{l, m, l-1}^{(1)}-u_{l, m, l+1}^{(1)}\right)\right]
\end{aligned}
$$

In order to derive the above equations, the Taylor expansion has been used:

$$
\begin{aligned}
u_{l \pm 1, m}(t) & =\sum_{j=1}^{\infty} \varepsilon^{j} u^{j}\left(\xi_{l} \pm \varepsilon a, \eta_{m}, \theta_{l \pm 1}, \tau\right) \\
& =\sum_{j=1}^{\infty} \varepsilon^{j} \sum_{s=0}^{\infty} \frac{1}{s !}\left[ \pm a \varepsilon \frac{\partial}{\partial \xi_{l}}\right]^{s} u_{l, m, l \pm 1}^{(j)} \\
u_{l, m \pm 1}(t) & =\sum_{j=1}^{\infty} \varepsilon^{j} u^{j}\left(\xi_{l}, \eta_{m} \pm \varepsilon b, \theta_{l}, \tau\right) \\
& =\sum_{j=1}^{\infty} \varepsilon^{j} \sum_{s=0}^{\infty} \frac{1}{s !}\left[ \pm b \varepsilon \frac{\partial}{\partial \eta_{m}}\right]^{s} u_{l, m, l}^{(j)}
\end{aligned}
$$

with

$$
\omega(k)=2 \sqrt{J_{2 x}} \sin \frac{k a}{2},
$$

where $U$ and $P$ are the real and complex functions, respectively, to be determined later, and $\bar{P}$ is its conjugate complex number.

When $j=2$ in (4), the second-order approximation equation is

$$
\begin{aligned}
\frac{\partial^{2}}{\partial t^{2}} & u_{l, m, l}^{(2)}-J_{2 x}\left(u_{l, m, l+1}^{(2)}-2 u_{l, m, l}^{(2)}+u_{l, m, l-1}^{(2)}\right) \\
= & -2 \mathrm{i} \omega\left(\lambda-\frac{a J_{2 x}}{\omega} \sin (k a)\right) \frac{\partial P}{\partial \xi_{l}} \exp \left(\mathrm{i} \theta_{l}\right) \\
& -8 \mathrm{i} J_{3 x} \sin (k a) \sin ^{2}\left(\frac{k a}{2}\right) P^{2} \exp \left(2 \mathrm{i} \theta_{l}\right)+\text { c.c. }
\end{aligned}
$$

where c.c. represents complex conjugate terms. In order to eliminate the secular term, we have

$$
\lambda=\frac{a J_{2 x}}{\omega} \sin (k a) .
$$

Then we can get the valid solution of (13) as follows:

$$
u_{l, m, l}^{(2)}=G_{0}\left(\xi_{l}, \eta_{m}, \tau\right)+G\left(\xi_{l}, \eta_{m}, \tau\right) \exp \left(\mathrm{i} \theta_{l}\right)+\mathrm{i} \frac{J_{3 x}}{J_{2 x}} c t g\left(\frac{k a}{2}\right) P^{2} \exp \left(2 \mathrm{i} \theta_{l}\right)+\text { c.c. }
$$

where $G_{0}$ and $G$ are, respectively, real and complex functions to be determined in higher-order approximations.

Considering $j=3$ in (4) and using the results obtained in (11), (12), (14) and (15), we can have

$$
\begin{aligned}
& \frac{\partial^{2}}{\partial t^{2}} u_{l, m, l}^{(3)}-J_{2 x}\left(u_{l, m, l+1}^{(3)}-2 u_{l, m, l}^{(3)}+u_{l, m, l-1}^{(3)}\right)=\left(J_{2 x} a^{2}-\lambda^{2}\right) \frac{\partial^{2} U}{\partial \xi_{l}^{2}}+b^{2} J_{2 y} \frac{\partial^{2} U}{\partial \eta_{m}^{2}}+8 a J_{3 x} \sin ^{2} \frac{k a}{2} \frac{\partial|P|^{2}}{\partial \xi_{l}} \\
& +\left[2 \mathrm{i} \omega \frac{\partial P}{\partial \tau}-\frac{a^{2} \omega^{2}}{4} \frac{\partial^{2} P}{\partial \xi_{l}^{2}}+b^{2} J_{2 y} \frac{\partial^{2} P}{\partial \eta_{m}^{2}}-2 \frac{J_{3 x}}{J_{2 x}} a \omega^{2} P \frac{\partial U}{\partial \xi_{l}}-\left(8 \frac{J_{3 x}^{2}}{J_{2 x}} \sin ^{2}(k a)+48 J_{4 x} \sin ^{4} \frac{k a}{2}\right)|P|^{2} P\right] \exp \left(\mathrm{i} \theta_{l}\right) \\
& + \text { c.c. + higher harmonics. }
\end{aligned}
$$


From (16) we find that there are two kinds of secular terms. The first ones are functions of the slow variables and make that $u_{l, m, l}^{(3)}$ contains $t$ explicitly. Removing these and the $\exp \left(\mathrm{i} \theta_{l}\right)$ secular terms, we obtain the two following equations concerning the envelope functions $U$ and $P$ :

$$
\begin{aligned}
& \frac{\partial^{2} U}{\partial \xi_{l}^{2}}+\kappa \frac{\partial^{2} U}{\partial \eta_{m}^{2}}+\mu \frac{\partial|P|^{2}}{\partial \xi_{l}}=0 \\
& \mathrm{i} \frac{\partial P}{\partial \tau}+\gamma \frac{\partial^{2} P}{\partial \xi_{l}^{2}}-\kappa \gamma \frac{\partial^{2} P}{\partial \eta_{m}^{2}}+\rho P \frac{\partial U}{\partial \xi_{l}}+\beta|P|^{2} P=0
\end{aligned}
$$

with

$$
\begin{aligned}
& \kappa=\frac{b^{2} J_{2 y}}{a^{2} J_{2 x}} \csc ^{2} \frac{k a}{2}, \quad \mu=\frac{8 J_{3 x}}{a J_{2 x}}, \\
& \gamma=-\frac{a^{2} \omega}{8}, \quad \rho=-\frac{a J_{3 x} \omega}{J_{2 x}} \\
& \beta=-\frac{4 \omega J_{3 x}^{2}}{J_{2 x}^{2}} \cos ^{2} \frac{k a}{2}-\frac{6 \omega J_{4 x}}{J_{2 x}} \sin ^{2} \frac{k a}{2}, \\
& \mu>0, \quad \kappa>0, \quad \gamma, \rho, \beta<0 .
\end{aligned}
$$

Using the transformation $P=\sqrt{\frac{-4}{\beta}} \frac{A}{\varepsilon}, \frac{\partial U}{\partial \xi_{l}}=\frac{-2 Q}{\rho \varepsilon^{2}}, x=$ $\frac{\xi_{l}}{\varepsilon \sqrt{-2 \gamma}}, y=\frac{\eta_{m}}{\varepsilon \sqrt{-2 \kappa \gamma}}$, (17) can be transformed into

$$
\begin{aligned}
& Q_{x x}+Q_{y y}+4|A|_{x x}^{2}=0, \\
& \mathrm{i} A_{t}-\frac{1}{2}\left(A_{x x}-A_{y y}\right)-2 A Q-4|A|^{2} A=0 \\
& \text { with } \mu \rho=2 \beta .
\end{aligned}
$$

We can find that (19) is just the DS-II [21 - 23]. It is relevant in many different physical contexts and describes slow modulation effects of a complex amplitude $A$, due to a small nonlinearity, on a monochromatic wave in a dispersive medium. It turns out to be integrable via the inverse spectral method [24-25]. Several direct methods to obtain special solutions of DS-II have been constructed, such as Darboux transformations [26], gauge transformations [27], $\bar{\partial}$ dressing method [28], Wronskian scheme [29-30].

\section{Periodic Solution, Soliton Solution and Rational Function Solution}

This section is devoted to solve the DaveyStewartson II equation, and then the leading order approximated solutions of the $2 \mathrm{D}$ monatomic lattice are got by explicit solutions of the DS-II.

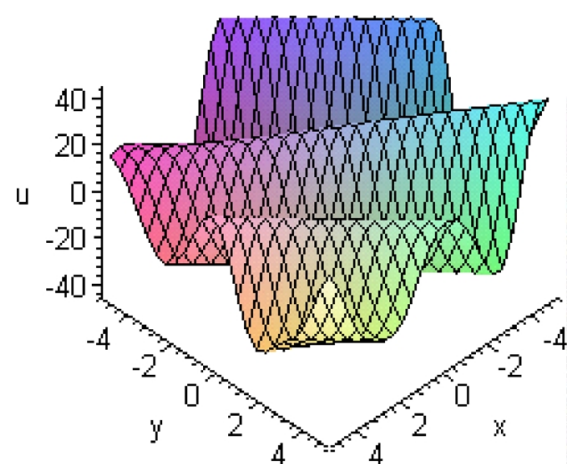

Fig. 2. Plot of solution (21) with $k_{1}=l_{1}, b_{2}=f=0$.

\subsection{Periodic Solutions}

It can be easily verified that (19) has periodic solutions of the form

$$
\begin{aligned}
Q= & b_{0}+b_{1} \sin \left(k_{1} x+l_{1} y+O_{1} t\right) \\
& +b_{2} \sin \left(2 k_{1} x+2 l_{1} y+2 O_{1} t\right) \\
& +b_{3} \cos \left(2 k_{1} x+2 l_{1} y+2 O_{1} t\right), \\
A= & \exp \left(\mathrm{i}\left(k_{0} x+l_{0} y+O_{0} t\right)\right) \\
& \cdot\left[c+d \sin \left(k_{1} x+l_{1} y+O_{1} t\right)\right. \\
& \left.+f \cos \left(k_{1} x+l_{1} y+O_{1} t\right)\right],
\end{aligned}
$$

where the coefficients satisfy $b_{1}=-4 c d, b_{0}=b_{2}=$ $f=0, b_{3}=d^{2}, k_{1}= \pm l_{1}, O_{0}=\frac{k_{0}^{2} d-4 d^{3}-8 d c^{2}-l_{0}^{2} d}{2 d}, O_{1}=$ $\pm k_{0} l_{1}-l_{0} l_{1}$, or $b_{0}=b_{1}=c=0, b_{2}=-2 f d, b_{3}=d^{2}-$ $f^{2}, k_{1}= \pm l_{1}, O_{0}=\frac{k_{0}^{2} d+2 d\left(d^{2}-f^{2}\right)-6 d^{3}-2 d f^{2}-l_{0}^{2} d}{2 d}, O_{1}=$ $\pm k_{0} l_{1}-l_{0} l_{1}$.

So, in the leading order approximation we can obtain the following periodic solution of the 2D monatomic lattice:

$$
\begin{gathered}
u(t)=\frac{\sqrt{-2 \gamma}}{\rho k_{1}}\left[2 b_{1} \cos \left(k_{1} x+l_{1} y+O_{1} t\right)\right. \\
+b_{2} \cos \left(2 k_{1} x+2 l_{1} y+2 O_{1} t\right) \\
\left.-b_{3} \sin \left(2 k_{1} x+2 l_{1} y+2 O_{1} t\right)\right] \\
+\left\{\sqrt { \frac { - 4 } { \beta } } \left[c+d \sin \left(k_{1} x+l_{1} y+O_{1} t\right)\right.\right. \\
\left.+f \cos \left(k_{1} x+l_{1} y+O_{1} t\right)\right]
\end{gathered}
$$

Figures 2 and 3 are the plots of the solution (21) at $t=2$ with $b_{2}=f=0, a=0.01, k=\frac{10 \pi}{3}, J_{2 x}=36$, 


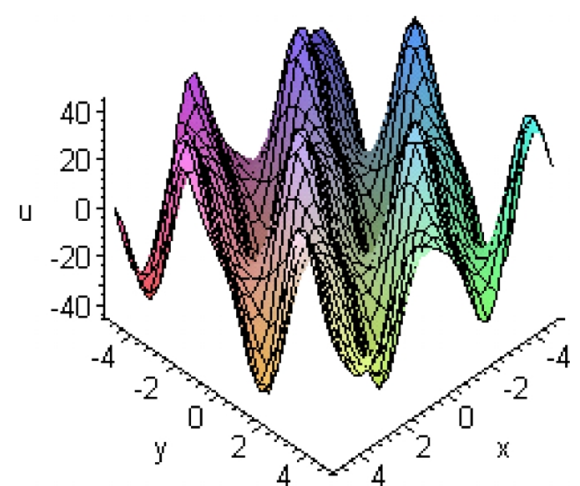

Fig. 3. Plot of solution (21) with $k_{1}=-l_{1}, b_{2}=f=0$.

$J_{3 x}=9, c=1, d=1.5, k_{0}=0.8, l_{0}=1, k_{1}= \pm l_{1}=$ \pm 1.2 . Figures 4 and 5 are the plots of the solution (21) at $t=2$ with $b_{1}=c=0, a=0.01, k=\frac{10 \pi}{3}, J_{2 x}=36$, $J_{3 x}=9, f=1.5, d=1.5, k_{0}=0.8, l_{0}=1, k_{1}= \pm l_{1}=$ \pm 1.2 .

\subsection{Soliton Solutions}

We find that (19) has the soliton solution

$$
\begin{aligned}
Q= & a_{0}+a_{1} \tanh \left(k_{1} x+l_{1} y+O_{1} t\right) \\
& +a_{2} \tanh \left(k_{1} x+l_{1} y+O_{1} t\right)^{2}, \\
A= & \exp \left(\mathrm{i}\left(k_{0} x+l_{0} y+O_{0} t\right)\right) \\
& \cdot\left[b_{0}+b_{1} \tanh \left(k_{1} x+l_{1} y+O_{1} t\right)\right],
\end{aligned}
$$

with $a_{0}=2 b_{1}^{2}-4 b_{0} b_{1}, a_{1}=-4 b_{0} b_{1}, a_{2}=-2 b_{1}^{2}, k_{1}=$ $\pm l_{1}, O_{0}=8 b_{0} b-4\left(b_{0}^{2}+b_{1}^{2}\right)+\frac{k_{0}^{2}-l_{0}^{2}}{2}, O_{1}= \pm k_{0} l_{1}-l_{0} l_{1}$.

So, in the leading order approximation we obtain the displacement of the 2D monatomic lattice as follows:

$$
\begin{aligned}
u(t)= & \frac{-2 \sqrt{-2 \gamma}}{\rho k_{1}} \\
& \cdot\left[a_{1} \operatorname{In}\left(\exp \left(k_{1} x+l_{1} y+O_{1} t\right)^{2}+1\right)\right. \\
& \left.+\frac{2 a_{2}}{\exp \left(k_{1} x+l_{1} y+O_{1} t\right)^{2}+1}\right] \\
& +\left[\sqrt{\frac{-4}{\beta}}\left[b_{0}+b_{1} \tanh \left(k_{1} x+l_{1} y+O_{1} t\right)\right]\right.
\end{aligned}
$$

Figure 6 is the plot of $Q$ at $t=2$ with

$$
\begin{array}{ll}
k_{0}=8, & l_{0}=-9, \quad k_{1}=l_{1}=-10, \\
b_{0}=1, & b_{1}=2,
\end{array}
$$

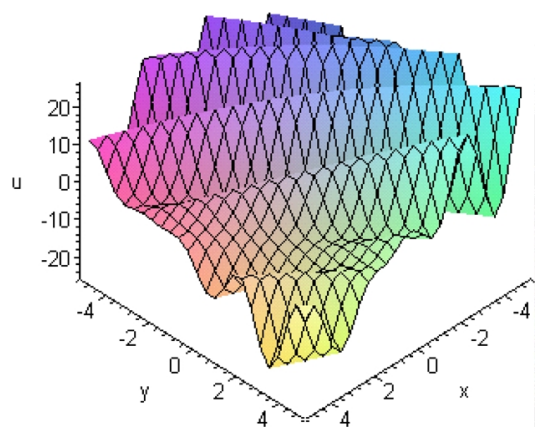

Fig. 4. Plot of solution (21) with $k_{1}=l_{1}, b_{1}=c=0$.

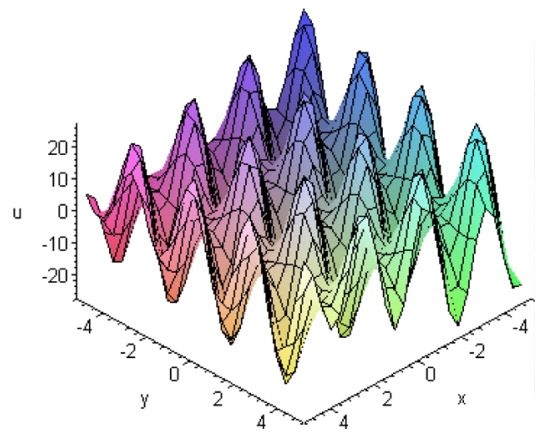

Fig. 5. Plot of solution (21) with $k_{1}=-l_{1}, b_{1}=c=0$.

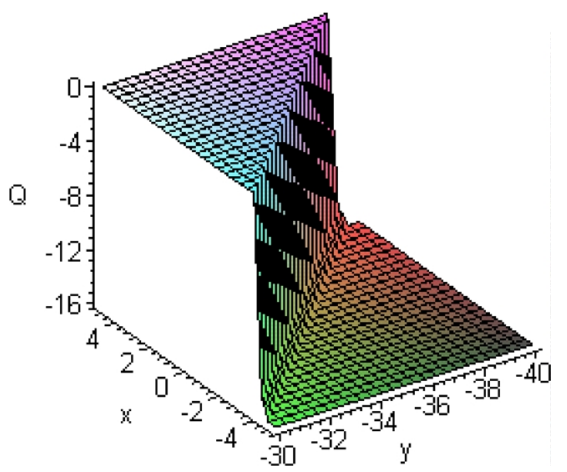

Fig. 6. Plot of $Q$ (22) with $a_{0}=0, a_{1}=a_{2}=-8, k_{1}=l_{1}=$ $-10, O_{1}=-170$.

from which we find that it is a kink grey soliton.

Figure 7 is the plot of the solution (23) at $t=2$ with

$$
\begin{aligned}
& a=0.01, \quad k=\frac{10 \pi}{3}, \quad J_{2 x}=36, \quad J_{3 x}=-9, \\
& k_{0}=8, \quad l_{0}=9, \quad k_{1}=l_{1}=-10 \\
& b_{0}=1, \quad b_{1}=2 .
\end{aligned}
$$

\subsection{Rational Solutions}

We now consider rational solutions of (19). Before solving them, we make the transformation: $Q=V_{x x}$, 


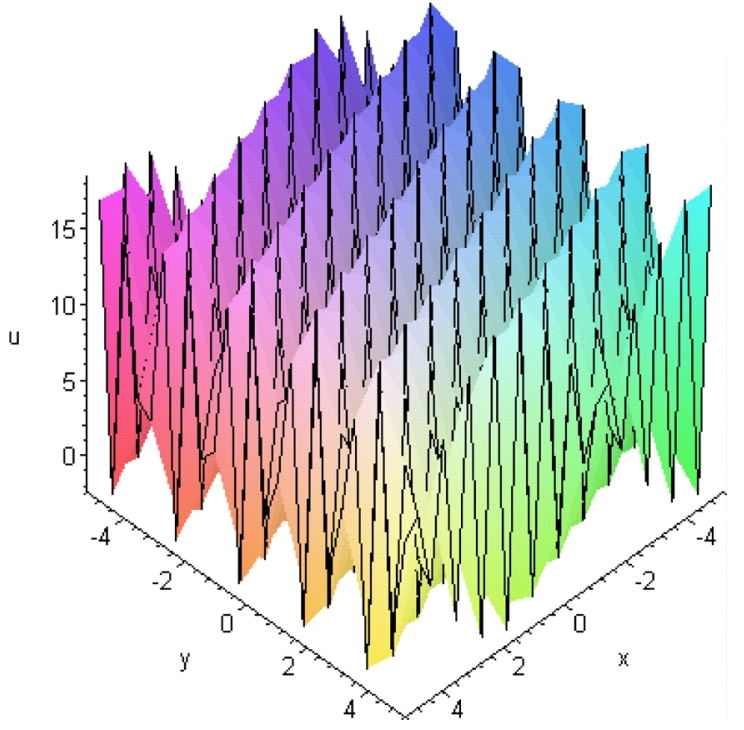

Fig. 7. Plot of solution (23) with $k_{1}=l_{1}$.

$z=x+\mathrm{i} y, \bar{z}=x-\mathrm{i} y$. Then (19) can be changed into

the form

$$
\begin{aligned}
& V_{z \bar{z}}+|A|^{2}=0, \\
& \mathrm{i} A_{t}-\left(A_{z z}+A_{\bar{z} \bar{z}}\right)-2 A\left(V_{z z}+V_{\bar{z} \bar{z}}\right)=0 .
\end{aligned}
$$

According to [29] and [30], the solution of the DS-II (24) can be expressed as follows:

$$
\begin{gathered}
A=\frac{\operatorname{det}\left(\begin{array}{ll}
w^{(1)} & w^{(2)} \\
\bar{w}^{(2)} & \bar{w}^{(1)}
\end{array}\right)}{\operatorname{det}\left(\begin{array}{ll}
w^{(1)} & w^{(2)} \\
\bar{w}^{(2)} & \bar{w}^{(1)}
\end{array}\right)}, \\
V=\ln \left(\operatorname{det}\left(\begin{array}{ll}
w^{(1)} & w^{(2)} \\
\bar{w}^{(2)} & \bar{w}^{(1)}
\end{array}\right)\right),
\end{gathered}
$$

where $\mathbf{w}^{(i)}(i=1,2)$ are $n \times n$ Wronskian matrices. $\hat{\mathbf{w}}^{(1)}$ and $\hat{\mathbf{w}}^{(2)}$ are also $n \times n$ matrices; the ${ }^{-}$represents conjugating every ingredient of the corresponding matrix, and the forms of the matrices $\mathbf{w}^{(1)}, \mathbf{w}^{(2)}, \hat{\mathbf{w}}^{(1)}$ and $\hat{\mathbf{w}}^{(2)}$ are

$$
\begin{array}{r}
\mathbf{w}^{(1)}=\left(\begin{array}{ccc}
\sigma_{1}^{(1)} & \ldots & \sigma_{n}^{(1)} \\
\partial \sigma_{1}^{(1)} & \ldots & \partial \sigma_{n}^{(1)} \\
\vdots & & \vdots \\
\partial^{n-1} \sigma_{1}^{(1)} & \ldots & \partial^{n-1} \sigma_{n}^{(1)}
\end{array}\right), \quad \mathbf{w}^{(2)}=\left(\begin{array}{ccc}
\sigma_{1}^{(2)} & \ldots & \sigma_{n}^{(2)} \\
\partial \sigma_{1}^{(2)} & \ldots & \partial \sigma_{n}^{(2)} \\
\vdots & & \vdots \\
\partial^{n-1} \sigma_{1}^{(2)} & \ldots & \partial^{n-1} \sigma_{n}^{(2)}
\end{array}\right), \\
\hat{\mathbf{w}}^{(1)}=\left(\begin{array}{ccc}
\sigma_{1}^{(1)} & \ldots & \sigma_{n}^{(1)} \\
\partial \sigma_{1}^{(1)} & \ldots & \partial \sigma_{n}^{(1)} \\
\vdots & & \vdots \\
\partial^{n-2} \sigma_{1}^{(1)} & \ldots & \partial^{n-2} \sigma_{n}^{(1)} \\
\bar{\partial}^{n} \bar{\sigma}_{1}^{(2)} & \ldots & \bar{\partial}^{n} \bar{\sigma}_{n}^{(2)}
\end{array}\right), \quad \hat{\mathbf{w}}^{(2)}=\left(\begin{array}{ccc}
\sigma_{1}^{(2)} & \ldots & \sigma_{n}^{(2)} \\
\partial \sigma_{1}^{(2)} & \ldots & \partial \sigma_{n}^{(2)} \\
\vdots & & \vdots \\
\partial^{n-2} \sigma_{1}^{(2)} & \ldots & \partial^{n-2} \sigma_{n}^{(2)} \\
\bar{\partial}^{n} \bar{\sigma}_{1}^{(1)} & \ldots & \bar{\partial}^{n} \bar{\sigma}_{n}^{(1)}
\end{array}\right),
\end{array}
$$

where $\sigma_{j}^{(i)}=\exp \left(\lambda_{j} z-\mathrm{i} \lambda_{j}^{2} t\right) f_{j}^{i}\left(z-2 \mathrm{i} \lambda_{j} t, t\right), \lambda_{j} \in \mathrm{C}, \bar{\sigma}_{j}^{(i)}$ is its conjugate complex number, $f_{j}^{i}$ is a linear combination of the polynomials $s_{n}(z, t)(n=1,2, \cdots)$, which can be constructed from $\exp \left(k z-\mathrm{i} k^{2} t\right)=\sum_{n \geq 0} s_{n}(z, t) k^{n} / n !$. We use the convenient notation $\partial=\partial / \partial z, \bar{\partial}=\partial / \partial \bar{z}$ with $z=x+\mathrm{i} y, \bar{z}=x-\mathrm{i} y$.

As in [30], we use the notation

$$
\left(n \mid m_{1}^{(1)}, \cdots, m_{n}^{(1)} ; m_{1}^{(2)}, \cdots, m_{n}^{(2)}\right)
$$

to indicate the corresponding family of solutions of (24), where $n$ is the rank of the Wronskian blocks and $m_{1}^{(1)}, \cdots, m_{n}^{(1)} ; m_{1}^{(2)}, \cdots, m_{n}^{(2)}$ are the leading degrees of the polynomials $f_{1}^{(1)}, \cdots, f_{n}^{(1)} ; f_{1}^{(2)}, \cdots, f_{n}^{(2)}$.

Example 1: Taking $(1 \mid 2 ; 0)$ in (27), corresponding to $f^{(1)}=s_{2}(z, t)+c s_{1}(z, t), f^{(2)}=1$, the solution of (24) reads

$$
\begin{aligned}
& A=\frac{4 \mathrm{i} \lambda_{1} t-2 z-c}{\left|\left(z-2 \mathrm{i} \lambda_{1} t\right)^{2}-2 \mathrm{i} t+c\left(z-2 \mathrm{i} \lambda_{1} t\right)\right|^{2}-1} \exp \left(\lambda_{1} z-\mathrm{i} \lambda_{1}^{2} t-\bar{\lambda}_{1} \bar{z}-\mathrm{i} \bar{\lambda}_{1}^{2} t\right), \\
& V=\ln \left[\left|\left(z-2 \mathrm{i} \lambda_{1} t\right)^{2}-2 \mathrm{i} t+c\left(z-2 \mathrm{i} \lambda_{1} t\right)\right|^{2}-1\right]+\lambda_{1} z-\mathrm{i} \lambda_{1}^{2} t+\bar{\lambda}_{1} \bar{z}+\mathrm{i} \bar{\lambda}_{1}^{2} t,
\end{aligned}
$$

where $z=x+\mathrm{i} y$, and $\bar{\lambda}_{1}$ is its conjugate complex number. 
Thus in the leading order approximation we obtain the rational solution of the $2 \mathrm{D}$ monatomic lattice as

$$
\begin{aligned}
u(t)= & \frac{-4 \sqrt{-2 \gamma} \operatorname{Re}\left\{\left[\left(z-2 \mathrm{i} \lambda_{1} t\right)^{2}-2 \mathrm{i} t+c\left(z-2 \mathrm{i} \lambda_{1} t\right)\right]\left[2\left(\bar{z}+2 \mathrm{i} \bar{\lambda}_{1} t\right)+\bar{c}\right]\right\}}{\rho\left[\left|\left(z-2 \mathrm{i} \lambda_{1} t\right)^{2}-2 \mathrm{i} t+c\left(z-2 \mathrm{i} \lambda_{1} t\right)\right|^{2}-1\right]}+\frac{-2 \sqrt{-2 \gamma}\left(\lambda_{1}+\bar{\lambda}_{1}\right)}{\rho} \\
& +\left(\sqrt{\frac{-4}{\beta}} \frac{4 \mathrm{i} \lambda_{1} t-2 z-c}{\left|\left(z-2 \mathrm{i} \lambda_{1} t\right)^{2}-2 \mathrm{i} t+c\left(z-2 \mathrm{i} \lambda_{1} t\right)\right|^{2}-1} \exp \left(2 \mathrm{i}\left[\operatorname{Im}\left(\lambda_{1} z\right)-\operatorname{Re}\left(\lambda_{1}^{2} t\right)\right]+\mathrm{i} \theta_{l}\right)+\text { c.c. }\right),
\end{aligned}
$$

where $z=x+\mathrm{i} y=\frac{l a-\lambda t}{\sqrt{-2 \gamma}}+\frac{\mathrm{i} m b}{\sqrt{-2 \kappa \gamma}}$, and $\bar{\lambda}_{1}$ and $\bar{c}$ are complex conjugates.

Example 2: Taking $(1 \mid 3 ; 0)$ in (27), corresponding to $f^{(1)}=s_{3}(z, t), f^{(2)}=1$, the solution of (24) reads

$$
\begin{aligned}
& A=\frac{3\left[-\left(z-2 \mathrm{i} \lambda_{1} t\right)^{2}+2 \mathrm{i} t\right] \exp \left(\lambda_{1} z-\mathrm{i} \lambda_{1}^{2} t-\bar{\lambda}_{1} \bar{z}-\mathrm{i} \bar{\lambda}_{1}^{2} t\right)}{\left|\left(z-2 \mathrm{i} \lambda_{1} t\right)^{3}-6 \mathrm{i} t\left(z-2 \mathrm{i} \lambda_{1} t\right)\right|^{2}-1}, \\
& V=\ln \left[\left|\left(z-2 \mathrm{i} \lambda_{1} t\right)^{3}-6 \mathrm{i} t\left(z-2 \mathrm{i} \lambda_{1} t\right)\right|^{2}-1\right]+\lambda_{1} z-\mathrm{i} \lambda_{1}^{2} t+\bar{\lambda}_{1} \bar{z}+\mathrm{i} \bar{\lambda}_{1}^{2} t
\end{aligned}
$$

where $z=x+\mathrm{i} y$, and $\bar{\lambda}_{1}$ is its conjugate complex number.

Then in the leading order approximation the displacement of the 2D monatomic lattice has the following form

$$
\begin{aligned}
u(t)= & \frac{-4 \sqrt{-2 \gamma} \operatorname{Re}\left\{\left[\left(z-2 \mathrm{i} \lambda_{1} t\right)^{3}-6 \mathrm{i} t\left(z-2 \mathrm{i} \lambda_{1} t\right)\right]\left[3\left(\bar{z}+2 \mathrm{i} \bar{\lambda}_{1} t\right)^{2}+6 \mathrm{i} t\right]\right\}}{\rho\left[\left|\left(z-2 \mathrm{i} \lambda_{1} t\right)^{3}-6 \mathrm{i} t\left(z-2 \mathrm{i} \lambda_{1} t\right)\right|^{2}-1\right]}+\frac{-2 \sqrt{-2 \gamma}\left(\lambda_{1}+\bar{\lambda}_{1}\right)}{\rho} \\
& +\left(\sqrt{\frac{-4}{\beta}} \frac{3\left[-\left(z-2 \mathrm{i} \lambda_{1} t\right)^{2}+2 \mathrm{i} t\right]}{\left|\left(z-2 \mathrm{i} \lambda_{1} t\right)^{3}-6 \mathrm{i} t\left(z-2 \mathrm{i} \lambda_{1} t\right)\right|^{2}-1} \exp \left(2 \mathrm{i}\left[\operatorname{Im}\left(\lambda_{1} z\right)-\operatorname{Re}\left(\lambda_{1}^{2} t\right)\right]+\mathrm{i} \theta_{l}\right)+\text { c.c. }\right),
\end{aligned}
$$

where $z=x+\mathrm{i} y=\frac{l a-\lambda t}{\sqrt{-2 \gamma}}+\frac{\mathrm{i} m b}{\sqrt{-2 \kappa \gamma}}$, and $\bar{\lambda}_{1}$ is its conjugate complex number.

\section{Conclusion}

By using the method of multiple scales combined with a quasidiscreteness approximation, we investigate the two-dimensional monatomic lattice with nearestneighbor interaction. From the original motion equation of the 2D lattice we obtain the Davey-Stewartson II equation, which is relevant in many physical contexts and describes slow modulation effects of the complex amplitude. Several direct methods have been proposed to solve the DS-II [26-30]. By solving the DS-II, we get explicit periodic solutions, soliton solutions and rational function solutions, and then the

[1] T. Qiang, Z. Hui, and Z. Rui, Sci. China, Ser. A 45, 884 (2002).

[2] T. Qiang and W. Changshu, Phys. Lett. 262A, 83 (1999).

[3] K. Nakkeeran, K. Porsezian, P. S. Sundaram, and A. Mahalingam, Phys. Rev. Lett. 80, 1425 (1998).

[4] P. Maniadis, G. P. Tsironis, A. R. Bishop, and A. V. Zolotaryuk, Phys. Rev. E 60, 7618 (1999).

[5] E. Fermi, J. Pasta, and S. Ulam, Los Alamos National leading order approximated solutions constructed by the explicit solutions of the DS-II are obtained as expressed in (21), (23), (29) and (31), some graphical representations of which are given in Figs. $2-7$.

\section{Acknowledgements}

The authors thank Professor Sen-yue Lou for his help and discussions. The project was supported by the Nature Science Foundation of Zhejiang Province under Grant No. Y604036, and the State Key Laboratory of Oil/Gas Reservoir Geology and Exploitation $\backslash$ PLN0402".

Laboratory Report LA1940,1955. Also in: Collected Papers of Enrico Fermi, University of Chicago Press, Chicago 1962, Vol. 2, p. 978.

[6] N. J. Zabusky and M. D. Kruskal, Phys. Rev. Lett. 15, 240 (1965).

[7] M. Remoissenet, Waves Called Solitons, 2nd ed., Springer-Verlag, Berlin 1996.

[8] G.-X. Huang, Z.-P. Shi, and Z.-X. Xu, Phys. Rev. B 47, 14561 (1993). 
[9] M. D. Krusal and N. J. Zabusky, Phys. Lett. 5A, 231 (1964).

[10] J. Satsuma and M. J. Ablowitz, J. Math. Phys. 20, 1496 (1979).

[11] R. Bourbonnais and R. Maynard, Phys. Rev. Lett. 64, 1397 (1990).

[12] V. M. Burlakov, S. A. Kiselev, and V. N. Pyrkov, Phys. Rev. B 42, 4921 (1990).

[13] I. Anders, V. Kotlyaroy, and E. Khruslov, Theor. Math. Phys. 99, 402 (1994).

[14] M. Tajiri, H. Miura, and T. Arai, Phys. Rev. E 66, 067601 (2002)

[15] T. Ioannidou, J. Pouget, and E. Aifantis, J. Phys. A: Math. Gen. 36, 643 (2003).

[16] A. Tsurui, Prog. Theor. Phys. 48, 1196 (1972).

[17] G.-X. Huang, Phys. Rev. B 51,12347 (1995).

[18] B. Hu, G.-X. Huang, and M. G. Velarde, Phys. Rev. E 62, 2827 (2000).

[19] V. V. Konotop, Phys. Rev. E 53, 2843 (1996).

[20] M. Remoissenet, Waves Called Solitons, SpringerVerlag, Berlin, Heidelberg, New York 1996, pp. 138 204.
[21] D. Davey and K. Stewartson, Proc. R. Soc. A 338, 101 (1974).

[22] M. J. Ablowitz and P. A. Clarkson, Solitons, Nonlinear Evolution Equations and Inverse Scattering, London Math. Soc. Lec. Not. Ser. 149, Cambridge University Press, Cambridge 1989.

[23] B. G. Konopelchenko, Solitons in Multidimensions. Inverse Spectral Transform Method, World Scientific, Singapore 1993.

[24] A. S. Fokas and M. J. Ablowitz, J. Math. Phys. 25, 2494 (1984).

[25] V. A. Arkadiev, A. K. Progrebkov, and M. C. Polivanov, Physica D 36, 189 (1989).

[26] S. B. Leble, M. A. Salle, and A. V. Yurov, Inverse Problems 8, 207 (1992).

[27] Y. Cheng, Y. S. Li, and G. X. Tang, J. Phys. A 23, L473 (1990).

[28] V. G. Dubrosky, J. Phys. A 32, 369 (1999).

[29] F. Guil and M. Mañas, J. Phys. A 28, 1713 (1995).

[30] M. Mañas and P. M. Santini, Phys. Lett. 227A, 325 (1997). 